Pacific Journal of Mathematic 


\section{QUADRATIC FORMS AND POWER SERIES FIELDS}

\section{LAWRENCE BERMAN}

The goal of this paper is to explore the connection between three properties of a field $F$ of characteristic not 2. Roughly speaking, these are:

(a) the Witt ring $W(F)$ is a group ring $R[G]$ with $G$ an Abelian group of exponent 2.

(b) the Witt ring $W(F)$ is isomorphic to $W(K)$ where $K$ is a power series field (i.e., $F$ is equivalent to $K$ with respect to quadratic forms).

(c) there are "enough" rigid elements in $F$.

Our purpose is to show that the connection is in some sense "quantitative", by showing that (a) and (b) can be "measured" by the index of a certain subgroup $A(F)$ in $\dot{F}$.

Preliminaries. (For proofs or details see [2] and [4]).

If $q$ is a quadratic form over $F$, then $D_{F}(q)$ consists of the elements in $\dot{F}=F-\{0\}$ which are represented by $q$. We denote by $\left\langle x_{1}, \cdots, x_{n}\right\rangle \cong q$ a diagonalization of an $n$-dimensional form $q$. The group $D_{F}(\langle 1, x\rangle), x \in \dot{F}$, may be seen as the nonzero image of the norm map from $F(\sqrt{-x})$ to $F$. If $D_{F}(\langle 1, b\rangle)=\dot{F}^{2} \cup b \dot{F}^{2}$ (as small as is possible) then we say that $b$ is rigid in $F$. Denote by $A(F)$ the set of all elements $x \in \dot{F}$ such that $x$ or $-x$ is nonrigid, together with the group $\dot{F}^{2} \cup-\dot{F}^{2}$. Then $A(F)$ is a subgroup of $\dot{F}$. Note that $b \notin A(F)$ if and only if $b$ and $-b$ are rigid nonsquares.

Recall that the Witt ring $W(F)$ is a ring whose elements are equivalence classes of nonsingular quadratic forms, where $q$ is equivalent to $q^{\prime}$, written $q=q^{\prime}$, if and only if $q \perp\langle-1\rangle q^{\prime}$ is hyperbolic. Each equivalence class is represented by a unique anisotropic form. To avoid cumbersome notation, it is customary to refer to a form $q \in W(F)$ and mean any form equivalent to $q$. By $q_{a n}$ we mean the unique anisotropic form $q_{a n}=q$. Set

$$
T(F)=\left\{q \in W(F) \mid D_{F}\left(q_{\text {an }}\right) \leqq A(F)\right\} .
$$

Then (see [2]), $T(F)$ is an excellent subring of $W(F)$ which is additively generated over $Z$ by the one-dimensional forms $\{\langle a\rangle \mid a \epsilon$ $A(F)\}$. Moreover

THEOREM $1.1[2] . \quad W(F) \cong T(F)[\dot{F} / A(F)]$. More precisely, if $\left\{b_{i} A(F), i \in I\right\}$ is a $\boldsymbol{Z} / 2 Z$-basis of $\dot{F} / A(F)$ and $B$ is the multiplicative subgroup of $W(F)$ generated by the unary forms $\left\{\left\langle b_{i}\right\rangle, i \in I\right\}$, then $W(F)=T(F)[B]$. 
(In general, if $G$ is a multiplicative group, and $\left\{g_{i}, i \in I\right\}$ a subset of $G$, we denote by $\left\langle\left\{g_{i}, i \in I\right\}\right\rangle$ the subgroup of $G$ generated by $\left\{g_{i}, i \in I\right\}$.)

REMaRK. The definition of $A(F)$ given in [2] differs slightly from that which we give here. It is not difficult to see that this does not affect the validity of the results there cited. We prefer the above definition since for the field $\boldsymbol{F}_{5}$ with five elements one obtains $A\left(\boldsymbol{F}_{5}\right)=\dot{\boldsymbol{F}}_{5}^{2} \varsubsetneqq \dot{\boldsymbol{F}}_{5}$, i.e., there are "enough" rigid elements. And, in fact, $W\left(\boldsymbol{F}_{5}\right)$ is a group ring.

Our motivation was an old result of T. A. Springer on fields of formal Laurent series [5] (see also [4, Ch. 6]). Specifically, let $F((x))$ denote such a field, with $F$-coefficients, which may be obtained as the quotient field of the ring of formal power series $F[[x]]$. We refer to $F((x))$ as a power series field.

THEOREM 1.2 [4]. Let $F$ be a field (of characteristic not 2). Then

(i) $W(F((x)))=W(F)[\{\langle 1\rangle,\langle x\rangle\}]$ (a group ring).

(ii) $x \notin A(F((x)))$ (power series fields have enough rigid elements).

In what follows we will consider fields produced by iterating the method of obtaining a power series field. One obtains thus fields of the type $F\left(\left(x_{1}\right)\right)\left(\left(x_{2}\right)\right) \cdots\left(\left(x_{n}\right)\right)$ which we denote $F\left\{\left(\left(x_{i}\right)\right), i=\right.$ $1, \cdots, n\}$. Let now $I$ be any set. We define the iterated power series field $K=F\left\{\left(\left(x_{i}\right)\right), i \in I\right\}$ as follows. First well-order $I$. Then $K$ is the union (composite) of the iterated power series fields of the form $F\left\{\left(\left(x_{j}\right)\right), j \in J\right\}$ where $J$ ranges over all finite (ordered) subsets of $I$ (with respect to the fixed ordering of $I$ ).

It is now possible to make precise the main results to be demonstrated in this paper, and to show what we meant above by "quantitative" equivalence:

A. Theorem 4.1. For any field $F$, if $W(F)=R[G]$ and $G$ has exponent 2, then there is a canonical injection of $G$ into $\dot{F} / A(F)$.

B. Corollary 3.2. For any field $F$, there is an extension field $k$ of $F$ with $\left.W(F) \cong W\left(k\left\{\left(\left(x_{i}\right)\right), \quad i \in I\right\}\right)=W(k)\left[\left\{\left\langle x_{i}\right\rangle, i \in I\right\}\right\rangle\right]$, where the cardinality of $I$ equals the $Z / 2 Z$-dimension of $\dot{F} \mid A(F)$.

This explains the terminology "enough rigid elements" in (c) and the "quantitative equivalence" we claimed. Namely, the group $\dot{F} / A(F)$ acts as a true measure of the extent to which $W(F)$ is a group ring over a group of exponent 2, as well as a measure of 
the equivalence of $F$ to a power series field, with respect to quadratic forms.

2. Rigid elements under quadratic extension. In this section we discuss the relation between $A(F)$ and $A(F(\sqrt{a})$ ) for general quadratic extensions $F(\sqrt{a})$ over $F$, and in particular, when $a \notin$ $A(F)$. In the particular case, it will be seen that $A(F)$ remains "invariant" under quadratic extension. I.e., if $b \notin A(F)$ and $K=$ $F(\sqrt{b})$ then $A(K)=A(F) \cdot \dot{K}^{2}$. We need a result found in [1]:

Lemma 2.1. Let $K=F(\sqrt{a}), a \in \dot{F}$. Then for any $x \in \dot{F}$,

$$
D_{K}(\langle 1, x\rangle) \cap \dot{F}=D_{F}(\langle 1, a x\rangle) \cdot D_{F}(\langle 1, x\rangle) .
$$

The next result also holds for general quadratic extensions:

Proposition 2.2. Let $K=F(\sqrt{a}), a \in \dot{F}$. Then $A(K) \cap \dot{F} \leqq$ $\{1, a\} A(F)$.

Proof. Let $x \in A(K) \cap \dot{F}$. Since $x$ or $-x$ is not rigid over $K$ and $\{ \pm 1\} \subseteq A(F)$, we may assume that $x$ is nonrigid over $K$. Let $z \in D_{K}(\langle 1, x\rangle)-\{1, x\} \dot{K}^{2}$. By the Norm Principle [4, p. 208], or by direct computation, if $N$ is the norm from $K$ to $F$, then $N(z) \in$ $D_{F}(\langle 1, x\rangle)$. Suppose $x \notin A(F)$. We will show that $x \in a \cdot A(F)$. Since $x \notin A(F), \quad x$ is rigid, i.e., $D_{F}(\langle 1, x\rangle)=\{1, x\} \cdot \dot{F}^{2}$, so $N(z) \in\{1, x\} \dot{F}^{2}$. If $N(z) \in x \dot{F}^{2}$, then since $N(\dot{K})=D_{F}(\langle 1,-a\rangle), x \in D_{F}(\langle 1,-a\rangle)$. So $a \in D_{F}(\langle 1,-x\rangle)$. But $x \notin A(F)$ implies that $-x$ is rigid also, so $a \in$ $\{1,-x\} \dot{F}^{2}$. Now if $a \in \dot{F}^{2}$, the proposition is trivially true. And if $a \in-x F^{2}$, then $x \in a\left(-\dot{F}^{2}\right) \subseteq a A(F)$, as was desired. The only other possibility is that $N(z) \in \dot{F}^{2}$.

By [4, p. 202], $N(z) \in \dot{F}^{2}$ implies that $z \in \dot{F} \cdot \dot{K}^{2}$; in fact, we may assume that $z \in \dot{F} \cap D_{K}(\langle 1, x\rangle)$. Since $x \in \dot{F}$, the last lemma applies, and we find that $z \in D_{F}(\langle 1, a x\rangle) \cdot D_{F}(\langle 1, x\rangle)$. If $a x$ is rigid, then $z \epsilon$ $\{l, a, x, a x\} \cdot \dot{F}^{2}$ ( $x$ is rigid). So $z \in\{1, x\} \cdot \dot{K}^{2}$, a contradiction. Hence, $a x$ cannot be rigid, in other words, $x \in a A(F)$.

A much better result can be obtained under the assumption that $a \notin A(F)$ :

THEOREM 2.3. Let $b \notin A(F), K=F(\sqrt{b})$. Then $A(K)=A(F)$. $\dot{K}^{2}$.

Proof. Let $x$ be nonrigid in $F$ and $z \in D_{F}(\langle 1, x\rangle)-\{1, x\} \dot{F}^{2}$. If $x$ is rigid in $K$, then $z \in D_{K}(\langle 1, x\rangle)=\{1, x\} \dot{K}^{2}$. By [4, p. 202], $\dot{K}^{2} \cap$ 
$\dot{F}=\{1, b\} \dot{F}^{2}$, so $z \in\{1, b, x, b x\} \dot{F}^{2}$. Thus, $z \in\{b, b x\} \dot{F}^{2}$, so $b \in D_{F}(\langle 1, x\rangle)$ (a group). This implies that $-x \in D_{F}(\langle 1,-b\rangle)=\{1,-b\} \dot{F}^{2}$ (as $b \notin$ $A(F),-b$ is rigid). So $x \in \pm \dot{K}^{2} \subseteq A(K)$ if $x$ is rigid in $K$. Hence in general, $x \in A(K)$. We have just shown that $A(F) \cdot \dot{K}^{2} \subseteq A(K)$.

In the light of the last proposition it suffices to show that $A(K) \subseteq F \cdot K^{2}$ to complete the proof. For if this holds, then $A(K) \subseteq$ $\dot{F} \cdot \dot{K}^{2} \cap A(K)=(A(K) \cap \dot{F}) \cdot \dot{K}^{2} \subseteq A(F) \cdot \dot{K}^{2}$. Extend $b \dot{F}^{2} \in \dot{F} / \dot{F}^{2}$ to a $Z / 2 Z$-basis of $\dot{F} / \dot{F}^{2}$, say $\left\{b \dot{F}^{2}\right\} \cup\left\{a_{j} \dot{F}^{2}, j \in J\right\}$. Since $-b$ is rigid, $D_{F}(\langle 1,-b\rangle)=N(\dot{K})=\{1,-b\} \dot{F}^{2}$, so by [3, Theorem 8] a $Z / 2 Z$-basis of $\dot{K} / \dot{K}^{2}$ is given by

$$
\left\{a_{j} \dot{K}^{2}, j \in J\right\} \cup\left\{z \dot{K}^{2}\right\}
$$

where $z$ is any element in $\dot{K}-\dot{F} \cdot \dot{K}^{2}$ (for such $z \notin \dot{F} \cdot \dot{K}^{2}, N(z) \notin \dot{F}^{2}$, in fact $\left.N(\boldsymbol{z}) \in-b \dot{F}^{2}\right)$.

Suppose $z \in \dot{K}-\dot{F} \cdot \dot{K}^{2}$ and $z$ is nonrigid. Then $\{1, z\} \dot{K}^{2} \varsubsetneqq D_{K}(\langle 1, z\rangle)$. We have seen above that $\dot{F} \cdot \dot{K}^{2}$ has index 2 in $\dot{K}$, so $D_{K}(\langle 1, z\rangle) \cap$ $\dot{F} \cdot \dot{K}^{2} \supsetneq \dot{K}^{2}$. Thus there exists some $y \in D_{K}(\langle 1, z\rangle) \cap \dot{F}$ with $y \notin \dot{K}^{2}$. So $-z \in D_{K}(\langle 1,-y\rangle)$. By the Norm Principle again, $N(-z) \in D_{F}(\langle 1,-y\rangle)$. But $-z \notin \dot{F} \cdot \dot{K}^{2}$, so $N(-z) \in-b \dot{F}^{2}$ and $-b \in D_{F}(\langle 1,-y\rangle)$. Finally, $y \in D_{F}(\langle 1, b\rangle)=\{1, b\} \dot{F}^{2}$ (b is rigid), i.e., $y \in \dot{K}^{2}$, which is a contradiction. So each $z \notin \dot{F} \cdot \dot{K}^{2}$ is rigid.

Since $T(F)$ is defined in terms of $A(F)$, it is not surprising to find that $T(F)$ is also "invariant" under a "rigid extension". This will be the key result in our proof of Theorem 3.1 below.

THEOREM 2.4. Let $b \notin A(F), K=F(\sqrt{b})$. If $r^{*}: W(F) \rightarrow W(K)$ is the map induced by the inclusion of $F \rightarrow K$, then the restriction of $r^{*}$ to $T(F)$ is a canonical isomorphism onto $T(K)$.

Proof. Note that by Theorem 2.3, $A(K)=A(F) \cdot \dot{K}^{2}$ so $T(K)$ is additively generated by forms $\{\langle a\rangle, a \in A(K)\}=\left\{r^{*}(\langle a\rangle), a \in A(F)\right\}$. Thus, it is clear that the map $r^{*}: W(F) \rightarrow W(K)$, induced by the inclusion $F \rightarrow K$, maps $T(F)$ onto $T(K)$, i.e., $r^{*}(T(F))=T(K)$. It suffices to show that the restriction of $r^{*}$ to $T(F)$ is injective. Suppose $\varphi$ is an anisotropic form in $T(F)$ and $r^{*}(\varphi)$ is isotropic. Then by a result of Scharlau [4, p. 200], there is an isometry

$$
\varphi \cong\langle 1,-b\rangle\langle x\rangle \perp \gamma,
$$

for some $\gamma \in W(F)$ and $x \in \dot{F}$. So $\{x,-b x\} \subseteq D_{F}(\varphi)$. But $\varphi \in T(F)$, so $\{x,-b x\} \subseteq D_{F}(\varphi) \subseteq A(F)$ (as $\varphi$ is anisotropic). Since $A(F)$ is a group, this implies that $-b \in A(F)$ so $b \in A(F)$, which contradicts the hypothesis. Hence, if $\varphi \in T(F), r^{*}(\varphi)$ is anisotropic if and only 
if $\varphi$ is anisotropic. So $\varphi=0$ if and only if $r^{*}(\varphi)=0$ for any $\varphi \in$ $T(F)$.

REMARK. Although $A(F)$ and $T(F)$ are invariants with respect to "rigid extensions" there does not exist a natural isomorphism of $W(F)$ and $W(K)$ for $K=F(\sqrt{b}), b \notin A(F)$. From the proof of 2.3 we have that

$$
\dot{F} /\{1, a\} A(F) \cong\left(\dot{F} \cdot \dot{K}^{2}\right) / A(K)
$$

canonically. Since $\left(\dot{F} \cdot \dot{K}^{2}\right) / A(K)$ is a subgroup of $\dot{K} / A(K)$ of index 2, $\dot{F} /\{1, a\} A(F)$ embeds into $\dot{K} / A(K)$ with the same index. So $\dot{F} / A(F) \cong \dot{K} / A(K)$ but this is not natural, as it depends on the choice of a $Z / 2 Z$-basis of $\dot{K} / A(K)$, specifically, on the choice of the basis element $z A(K)$ where $z \in \dot{K}-\dot{F} \cdot \dot{K}^{2}$.

CoRollary 2.6. Let $F$ be a field, $b \notin A(F)$ and $K=F(\sqrt{b})$. Then $W(F)$ and $W(K)$ are ring isomorphic (not canonically).

Proof. We have $T(K) \cong T(F)$ and $\dot{K} / A(K) \cong \dot{F} / A(F)$. So by Theorem 1.2, $W(F) \cong W(K)$.

3. Finding power series fields. We are now prepared to construct a field $k \supseteqq F$ such that $W(k) \cong T(F)$ for any field $F$. Once this is accomplished, we can adjoin to $k$ the requisite number of iterated power series variables, to produce a field $K$ such that $W(K) \cong W(F)$. Specifically, the number of iterated power series variables we must adjoin to $k$ is equal to the $Z / 2 Z$-dimension of $\dot{F} / A(F)$. That is, if $\dot{F} / A(F)$ has a basis $\left\{b_{i} A(F) \mid i \in I\right\}$, then we will take $K=k\left\{\left(\left(x_{i}\right)\right), i \in I\right\}$.

Now such a field $K$ is the union of power series fields $K_{J}=$ $k\left\{\left(\left(x_{j}\right)\right), j \in J\right\}$, where $J$ ranges over all finite ordered subsets of $I$ (which has been well-ordered). By inductively applying Theorem 1.2 , we find that

$$
W\left(K_{J}\right)=W(k)\left[\left\langle\left\{\left\langle x_{j}\right\rangle, j \in J\right\}\right\rangle\right] .
$$

Since any form in $W(K)$ may be represented by a diagonalization whose entries lie in some $K_{J}, J$ finite,

$$
\begin{gathered}
W(K)=U\left(W\left(K_{J}\right) \bigotimes_{k} K\right) \\
J \text { finite } \subset I .
\end{gathered}
$$

In fact, we may identify $W\left(K_{J}\right)$ with its image $\left(W K_{J}\right) \bigotimes_{k} K$. For suppose $\varphi \in W\left(K_{J}\right)$ is anisotropic and $\varphi \otimes K$ is isotropic. Then also 
$\varphi \otimes K_{J^{\prime}}$ is isotropic, where $I \supseteq J^{\prime} \supseteqq J$ and $J^{\prime}$ is finite. Since $K_{J^{\prime}}=$ $K_{J}\left\{\left(\left(x_{j}\right)\right), j \in J^{\prime}-J\right\}$ and $J^{\prime}-J$ is finite, by again applying Theorem 1.2, we have that the $\operatorname{map} \varphi \rightarrow \varphi \otimes K_{J}$ takes anisotropic forms to anisotropic forms. So $\varphi \otimes K$ must be anisotropic. Using this identification

$$
\begin{aligned}
W(K)= & \cup W\left(k\left\{\left(\left(x_{j}\right)\right), j \in J\right\}\right) \\
& J \text { finite } \leqq I \\
= & \cup W(k)\left[\left\langle\left\{\left\langle x_{j}\right\rangle, j \in J\right\}\right\rangle\right] \\
& J \text { finite } \subseteq I \\
= & W(k)\left[\left\langle\left\{\left\langle x_{i}\right\rangle, i \in I\right\}\right\rangle\right] .
\end{aligned}
$$

Thus, if $k$ is chosen so that $W(k) \cong T(F)$, then

$$
W(K) \cong T(F)\left[\left\langle\left\{\left\langle x_{i}\right\rangle, i \in I\right\}\right\rangle\right] \cong T(F)[\dot{F} / A(F)]=W(F) .
$$

Recall that a 2-extension of a field $F$ is a field extension $k$ of $F$ which lies in a quadratic closure of $F$.

TheOREM 3.1. For any field $F$, there exists a field $k$ extending $F$ (a 2-extension) such that

(i ) $A(K)=\dot{k}=A(F) \cdot \dot{k}^{2}$,

(ii) $W(k) \cong T(F)$.

Corollary 3.2. Let $F$ be any field. Then there exists a field $K$ of iterated power series over a 2-extension $k$ of $F$ such that

(i) the number of power series variables is equal to the cardinality of a set $I$ indexing a $Z / 2 Z$-basis of $\dot{F} / A(F)$.

(ii) $W(F) \cong W(K)=W(k)\left[\left\langle\left\{\left\langle x_{i}\right\rangle, i \in I\right\}\right\rangle\right]$.

Proof of 3.1. Let $\mathscr{F}$ denote the set of all 2-extensions $L$ of $F$ in a fixed quadratic closure of $F$ with the following properties:

(1) $A(L)=A(F) \cdot L^{2}$,

(2) if $r_{L / F}^{*}: W(F) \rightarrow W(L)$ is the canonical map induced by the inclusion $F \rightarrow L$ then $r_{L / F}^{*}$ restricted to $T(F)$ is an injection, i.e., $T(F) \bigotimes_{F} L \cong T(F)$ via $r_{L / F}^{*}$.

Let us observe that $F \in \mathscr{F}$, so $\mathscr{F}$ is a nonempty set.

Suppose $\left\{L_{\beta}, \beta \in B\right\}$ is a chain in $\mathscr{F}$ (totally ordered by inclusion) and $L=\cup\left\{L_{\beta}, \beta \in B\right\}$. We claim that $L \in \mathscr{F}$. First note that (2) is clear. For suppose that $\varphi \in T(F)$ is anisotropic and $\varphi \bigotimes_{F} L=$ 0 . Then the $L$-isometry of $\varphi \bigotimes_{F} L$ and a hyperbolic form is effected by invertible matrices of finite size with coefficients in $L$. Since $L=\cup\left\{L_{\beta}, \beta \in B\right\}$, these matrices actually have coefficients in some $L_{\beta}$, where $\beta \in B$. I.e., for that $\beta \in B, \phi \bigotimes_{F} L_{\beta}=0$. But $r_{L_{\beta^{\prime} F}}^{*}$ injects $T(F)$ into $W\left(L_{\beta}\right)$, so $\varphi=0$ and (2) holds for $L$. 
Next, we need to show that $A(L)=A(F) \cdot \dot{L}^{2}$. Let $z \in A(L)$. We may assume that $z$ is not rigid since both $A(L)$ and $A(F)$ contain -1. Take $y \in D_{L}(\langle 1, z\rangle\} \dot{L}^{2}-\{1, z\} \dot{L}^{2}$, and write $y=x_{1}^{2}+x_{2}^{2} z$ where $x_{1}$, $x_{2} \in \dot{L}$. Now choose $\beta \in B$ such that $\left\{x_{1}, x_{2}, z\right\} \subseteq \dot{L}_{\beta}$. Clearly $y \notin\{1, z\}$. $\dot{L}_{\beta}^{2} \subseteq\{1, z\} \dot{L}^{2}$, so since $y \in D_{L_{\beta}}(\langle 1, z\rangle), z \in A\left(L_{\beta}\right)=A(F) \cdot \dot{L}_{\beta}^{2} \cong A(F) \cdot \dot{L}^{2}$. Conversely, take $x \in A(F), x \notin \pm \dot{F}^{2}$ and choose $x^{\prime} \in D_{F}(\langle 1, x\rangle)-\{1, x\}$. $\dot{F}^{2}$ (we may assume $x$ is nonrigid). Then $x^{\prime} \in D_{L}\left(\langle 1, x\rangle \bigotimes_{F} L\right)-\{1$, $x\} \dot{L}^{2}$. For if $x^{\prime} \in\{1, x\} \dot{L}^{2}$, then $\left\langle 1,-x^{\prime}\right\rangle \boldsymbol{\bigotimes}_{F} L$ or $\left\langle 1,-x x^{\prime}\right\rangle \boldsymbol{\bigotimes}_{F} L$ is hyperbolic. But $\left\langle 1,-x^{\prime}\right\rangle$ and $\left\langle 1,-x x^{\prime}\right\rangle$ are in $T(F)$ since $x^{\prime}$ (and $x$ ) is in $A(F)$. (Since $x \notin \pm \dot{F}^{2},-x \in D_{F}\left(\left\langle 1,-x^{\prime}\right\rangle\right)-\left\{1,-x^{\prime}\right\} \dot{F}^{2}$, so $-x^{\prime} \in$ $A(F)$.) So by (2), $\left\langle 1,-x^{\prime}\right\rangle$ or $\left\langle 1,-x x^{\prime}\right\rangle$ is $F$-hyperbolic; thus $x^{\prime} \in$ $\{1, x\} \dot{F}^{2}$, a contradiction. Hence $x \in A(L)$, and so $A(L)=A(F) \cdot \dot{L}^{2}$.

By Zorn's lemma, we may pick a maximal element $k \in \mathscr{F}$. We need only show, to conclude the proof, that $r_{k \mid F}^{*}$ maps $T(F)$ onto $W(k)$. This will be clear if we prove that $A(k)=\dot{k}$. For then, $T(k)=$ $W(k)$. Combining this with the fact that $A(k)=A(F) \cdot \dot{k}^{2}$ we have that $T(k)=T(F) \bigotimes_{F} k \cong T(F)$, so $W(k) \cong T(F)$ via $r_{k \mid F}^{*}$. Suppose, by way of contradiction, that $z \in \dot{k}-A(k)$. Let $k_{0}=k(\sqrt{z})$. Then by Theorem 2.3 we have $A\left(k_{0}\right)=A(k) \cdot \dot{k}_{0}^{2}=A(F) \cdot \dot{k}^{2} \cdot \dot{k}_{0}^{2}=A(F) \cdot \dot{k}_{0}^{2}$. Also, by Theorem 2.4, $r_{k_{0} / F}^{*}$ is an injection on $T(k)$. Since $r_{k ! F}^{*}$ is an isomorphism of $T(F)$ onto $T(k)$, the composition $r_{k_{0} \mid F^{\prime}}^{*}=r_{k_{0} \mid k}^{*} \circ r_{k \mid F}^{*}$ is an injection of $T(F)$ into $W\left(k_{0}\right)$. I.e., $k_{0} \in \mathscr{F}$, contradicting the maximality of $k$ among all 2 -extensions of $F$. Thus, $A(k)=\dot{k}$ and $W(k) \cong T(F)$.

REMARK. It is possible to give a more constructive proof of (3.1). Say a finite 2-extension $K / F$ is "admissible" if

$$
K=F_{n} \supseteqq F_{n-1} \supseteqq \cdots \supseteqq F_{1} \supseteqq F_{0}=F
$$

are quadratic extensions, $F_{i+1}=F_{i}\left(\sqrt{a_{i}}\right)$, with $a_{\imath} \in \dot{F}_{i}-A\left(F_{i}\right)$. Then by 2.3 and 2.4 we have inductively that $A(K)=A(F) \cdot \dot{K}^{2}$ and $T(K)=T(F) \bigotimes_{F} K \cong T(F)$.

Let $k$ be the composite of all admissible extensions within a fixed algebraic closure. Then we claim that $A(k)=A(F) \cdot \dot{k}^{2}=\dot{k}$ and $W(k)=T(k)=T(F) \boldsymbol{\otimes}_{F} k \cong T(F)$. Observe that if $x \in \dot{k}$, then $x$ lies in a composite $K=K_{1} \cdot K_{2} \cdots K_{r}$ where each $K_{i}$ is admissible, so also $K$ is admissible. The proofs that $A(k)=A(F) \cdot \dot{k}^{2}$ and that $T(F) \cong$ $T(F) \bigotimes_{F} k=T(k)$ all follow by arguments similar to those used in (3.1). All that remains then is to show, as above, that $\dot{k}=A(k)$. But if $x \in \dot{k}-A(k)$, then $x \in \dot{K}-A(K)$ for some admissible $K$ over $F$ (since $\left.A(K)=A(F) \cdot \dot{K}^{2} \cong A(F) \cdot \dot{k}^{2}=A(k)\right)$. So $K(\sqrt{x})$ is admissible. Thus $K(\sqrt{x}) \subseteq k$, so $x \in \dot{k}^{2} \subseteq A(k)$, a contradiction. Thus, $\dot{k}=A(k)$, so $T(F) \cong W(k)$. 
4. Group rings and rigid elements. The point of Theorem 1.1 is that when one knows the 2-group $\dot{F} / A(F)$, one also knows that $W(F)$ is a group ring over this same group. In this section we answer the opposite question: does knowing that $W(F)=R[G]$ for a 2-group $G$ tell us anything about $\dot{F} / A(F)$ ? Our main result is:

THEOREM 4.1. For any field $F$, if $W(F)=R[G]$ and $G$ is a 2-group, then there is a canonical injection det: $G \rightarrow \dot{F} / A(F)$.

In the case that $|G|=2$, this result was already demonstrated in [2]. Since we will make use of this, we produce it here:

THEOREM 4.2. Let $F$ be a field.

(i) If $W(F)=R[\langle 1\rangle,\langle x\rangle]$ for some $x \in \dot{F}$, then $x \notin A(F)$.

(ii) If $W(F)=R[G]$ and $|G|=2$, then $W(F)=R[\langle 1\rangle,\langle b\rangle]$ for some $b \in \dot{F}$. In fact, by (i), $b \notin A(F)$, so $G$ injects into $\dot{F} / A(F)$.

Now it is possible, when $|G|=2^{n}<\infty$ to prove 4.1 by induction on $n$. Namely, one writes $G=X_{i=1}^{n}\left\{\langle 1\rangle, q_{i}\right\}$ in terms of a $\boldsymbol{Z} / 2 \boldsymbol{Z}$ basis $\left\{q_{1}, \cdots, q_{n}\right\}$. Set $H_{1}=X_{i=2}^{n}\left\{\langle 1\rangle, q_{i}\right\}$. Then by $4.2, W(F)=$ $\left(R\left[H_{1}\right]\right)\left[\left\{\langle 1\rangle, q_{1}\right\}\right]=\left(R\left[H_{1}\right]\right)\left[\left\{\langle 1\rangle,\left\langle b_{1}\right\rangle\right\}\right]$ with $b_{1} \notin A(F)$. Next set $H_{2}=$ )$\left.X_{1=3}^{n}\left\{\langle 1\rangle, q_{i}\right\}\right) \times\left\{\langle 1\rangle\right.$, $\left.\left\langle b_{1}\right\rangle\right\}$, so $W(F)=\left(R\left[H_{2}\right]\right)\left[\left\{\langle 1\rangle, q_{2}\right\}\right]=R\left[H_{2}\right]\left[\left\{\langle 1\rangle,\left\langle b_{2}\right\rangle\right\}\right]$ with $b_{2} \notin A(F)$. Continuing in this fashion we obtain elements $\left\{b_{1}, \cdots, b_{n}\right\}$ all not in $A(F)$ such that if $\widetilde{G}=X_{i=1}^{n}\left\{\langle 1\rangle,\left\langle b_{i}\right\rangle\right\}, W(F)=$ $R[\widetilde{G}]$. Then fact that $W(F)$ is a free $R$-module on $|G|$-generators implies that $|\breve{G}|=|G|=2^{n}$, so $G$ and $\breve{G}$ are isomorphic as $Z / 2 Z$ vector spaces. This implies that $\left\{b_{1}, \cdots, b_{n}\right\}$ represent independent cosets of $A(F)$ in $\dot{F}$ (otherwise 4.2 (i) would give a contradiction). So there is an obvious injection of $\breve{G}$ into $\dot{F} / A(F)$. However we have not obtained a canonical injection of $G$ into $\dot{F} / A(F)$. We summarize this result:

COROLLARY 4.3. Let $F$ be a field and $W(F)=R[G]$ where $|G|=2^{n}<\infty$. Then there exists a multiplicative subgroup $\breve{G}$ of $W(F)$ such that

(i) $|\widetilde{G}|=2^{n}$.

(ii) $W(F)=R[\breve{G}]$.

(iii) $\widetilde{G}$ is generated by certain one-dimensional forms $\left\{\left\langle b_{i}\right\rangle, i=\right.$ $1, \cdots, n\}$ with $\left\{b_{1}, \cdots, b_{n}\right\} \subseteq \dot{F}$ and independent modulo $A(F)$.

(iv) $\breve{G}$ injects naturally into $\dot{F} / A(F)$.

In a sense, 4.1 is weaker than 4.3 , in that one cannot in general produce a group $\widetilde{G}$ satisfying (ii) and (iii) above. Clearly, the 
inductive argument fails if $|G|$ is not finite. However, by means of another approach it is possible to show that there is always a canonical injection of $G$ into $\dot{F} / A(F)$.

In order to do this, we consider first the ordinary determinant det: $\bar{W}(F) \rightarrow \dot{F} / \dot{F}^{2}$. Since $\operatorname{det}(\langle 1,-1\rangle)=-\dot{F}^{2}$, the map det does not induce a group homomorphism det: $W(F) \rightarrow \dot{F} / \dot{F}^{2}$. But by considering a "coarser" invariant $\overline{\operatorname{det}}: W(F) \rightarrow \dot{F} / \pm \dot{F}^{2}$, namely $\overline{\operatorname{det}}(q)=$ $\pm \operatorname{det}(q) \dot{F}^{2}$, one obtains an additive group homomorphism on $W(F)$. An even coarser invariant d $\widetilde{\text { et: }} W(F) \rightarrow \dot{F} / A(F)$ may be constructed by setting $\tilde{\operatorname{det}}(q)=\operatorname{det}(q) \cdot A(F)$ (note that $\pm \dot{F}^{2} \subseteq A(F)$ ).

Let us note that if $q_{1}$ and $q_{2}$ are odd-dimensional quadratic forms, then $\operatorname{det}\left(q_{1} \otimes q_{2}\right)=\operatorname{det}\left(q_{1}\right) \cdot \operatorname{det}\left(q_{2}\right)$. A similar equality holds for det. We next consider the group $G$ in 4.1 ; if $q \in G$, then $q^{2}=$ $q \otimes q=\langle 1\rangle$, so $q$ is odd-dimensional. Consequently, $\tilde{\text { det: }} G \rightarrow \dot{F} / A(F)$ is a multiplicative homomorphism.

Proof of 4.1. By the above discussion, it suffices to show that det: $G \rightarrow \dot{F} / A(F)$ is injective

We may clearly assume that $G$ is a nontrivial group. For each $q \in G, q \neq\langle 1\rangle$, we will define a homomorphism $\tau_{q}$ from $\dot{F}$ into the additive group $Z / 2 Z$. Fix one such $q \in G, q \neq\langle 1\rangle$. Choose some subring $S$ of $W(F)$ such that $W(F)=S[\{\langle 1\rangle, q\}]$. If $x \in \dot{F}$ and $\langle x\rangle=$ $s_{1}+s_{2} q$ with $s_{1}, s_{2} \in S$, define $\tau_{q}(x)=\operatorname{dim}_{0}\left(s_{2}\right)$ (recall that $\operatorname{dim}_{0}\left(s_{2}\right)=0$ if $\operatorname{dim}\left(s_{2}\right)$ is even and $=1$ if $\operatorname{dim}\left(s_{2}\right)$ is odd). As in the proof of 4.2 (ii) (see [2]), if $\tau_{q}(x)=1$, then $s_{2}$ is a unit in $W(F)$, and moreover $W(F)=S[\{\langle 1\rangle,\langle x\rangle\}]$. So if $\tau_{q}(x)=1$, then by 4.2 (i), $x \notin A(F)$. Thus, $A(F) \subseteq \operatorname{ker}\left(\tau_{q}\right)$. Observe that $\tau_{q}: \dot{F} \rightarrow Z / 2 Z$ is a homomorphism, for if $\langle x\rangle=s_{1}+s_{2} q$ and $\left\langle x^{\prime}\right\rangle=s_{1}^{\prime}+s_{2}^{\prime} q$, then $\left\langle x x^{\prime}\right\rangle=\left(s_{1} s_{1}^{\prime}+\right.$ $\left.s_{2} s_{2}^{\prime}\right)+\left(s_{1} s_{2}^{\prime}+s_{2} s_{1}^{\prime}\right) q$ (since $q^{2}=\langle 1\rangle$ ). And $\operatorname{dim}_{0}\left(s_{1} s_{2}^{\prime}+s_{2} s_{1}^{\prime}\right)=1$ if and only if $\operatorname{dim}_{0}\left(s_{2}\right) \neq \operatorname{dim}_{0}\left(s_{2}^{\prime}\right)$, so $\tau_{q}\left(x x^{\prime}\right)=\tau_{q}(x)+\tau_{q}\left(x^{\prime}\right)$.

We wish to show for any $q \in G, q \neq\langle 1\rangle$, that $\operatorname{det} q \notin A(F)$. Thus it will be enough to show that $\tau_{q}(\operatorname{det} q) \neq 0$, as $A(F) \subseteq \operatorname{ker}\left(\tau_{q}\right)$. Consider a diagonalization $\left\langle x_{1}, \cdots, x_{n}\right\rangle \cong q$. We have, in $Z / 2 Z$,

$$
\tau_{q}(\operatorname{det} q)=\tau_{q}\left(\prod_{i=1}^{n} x_{i}\right)=\sum_{i=1}^{n} \tau_{s}\left(x_{i}\right) .
$$

Write now, for each $i=1, \cdots, n$,

$$
\left\langle x_{i}\right\rangle=s_{i}+s_{i}^{\prime} q, s_{i}, s_{i}^{\prime} \in S
$$

(where $S$ is the subring of $W(F)$ used to define $\tau_{q}$ ). Then

$$
q=\sum_{i=1}^{n}\left\langle x_{i}\right\rangle=\left(\sum_{i=1}^{n} s_{i}\right)+\left(\sum_{i=1}^{n} s_{i}^{\prime}\right) q,
$$


so by the group ring property, $\sum_{i=1}^{n} s_{i}^{\prime}=\langle 1\rangle$. Hence, in $Z / 2 Z$,

$$
1=\operatorname{dim}_{0}\left(\sum_{i=1}^{n} s_{i}^{\prime}\right)=\sum_{i=1}^{n} \operatorname{dim}_{0}\left(s_{i}^{\prime}\right)=\sum_{i=1}^{n} \tau_{q}\left(x_{i}\right) .
$$

Hence, $\tau_{q}(\operatorname{det} q)=1$, so $\operatorname{det} q \notin A(F)$, and $\tilde{\operatorname{det}}: G \rightarrow \dot{F} / A(F)$ is injective.

Note. The definition of $\tau_{q}$ in the above proof is not independent of the choice of a subring $S \subseteq W(F)$ satisfying $W(F)=$ $S[\{\langle 1\rangle, q\}]$. For example, let $F=\boldsymbol{C}((x))((y))$ and take $S=W(\boldsymbol{C}((x)))$, $S^{\prime}=W(\boldsymbol{C}((x y)))$. Then

$$
W(F)=S[\{\langle 1\rangle,\langle y\rangle\}]=S^{\prime}[\{\langle 1\rangle,\langle y\rangle\}] .
$$

So on the one hand, with respect to $S$, we have $\tau_{\langle y\rangle}(x)=0$ as $\langle x\rangle \in S$. And on the other hand, with respect to $S^{\prime}$, we have $\tau_{\langle y\rangle}^{\prime}(x)=$ 1 as $\langle x\rangle=\langle x y\rangle \cdot\langle y\rangle$ and $\langle x y\rangle \in S^{\prime}$.

If $2 \neq 0$ in $W(F)$ (i.e., $-1 \notin \dot{F}^{2}$ ) then (4.3) may be put in a simpler form:

Corollary 4.4. Let $F$ be a field with $-1 \notin \dot{F}^{2}$. If $W(F)=$ $R[G]$ where $G$ is a finite group of exponent 2, then $W(F)=R[\widetilde{G}]$ where $\widetilde{G}$ consists of unary forms $\left\{\left\langle b_{q}\right\rangle, q \in G\right\}$ with $\operatorname{det} q=b_{q} A(F)$.

Proof. For a given $q \in G-\langle 1\rangle$, we may decompose, via Theorem 1.1:

$$
q=\sum_{i=1}^{k}\left\langle b_{i}\right\rangle \varphi_{i},
$$

where each $\varphi_{i} \in T(F)$ and $\left\{b_{1}, \cdots, b_{n}\right\}$ represent distinct cosets of $A(F)$ in $\dot{F}$. As $q^{2}=\langle 1\rangle$, whenever $1 \leqq i<j \leqq k, 2 \varphi_{i} \varphi_{j}=0$. Since $q$ is odd-dimensional, some $\varphi_{i}$, say $\varphi_{1}$, is odd-dimensional, so is not a zero-divisor $\left[4\right.$, p. 250]. Hence, $2 \varphi_{i}=0$ for $i=2, \cdots, k$. So since $2 \neq 0$, each $\varphi_{i}, i>1$, is even-dimensional. Now each $\varphi_{i}$ may be diagonalized by elements of $A(F)$, so for each $i, \operatorname{det}\left(\left\langle b_{i}\right\rangle \varphi_{i}\right)=b_{i}^{d_{i}}$. $A(F)$, where $d_{i}=\operatorname{dim}_{0}\left(\varphi_{i}\right)$. Hence $\tilde{\operatorname{det}}(q)=b_{1} A(F)$. Set $b_{q}=b_{1}$. Observe that $b_{q} \notin A(F)$. In the proof given above of 4.3 if we replace each generator $q$ of $G$ with the unary form $\left\langle b_{q}\right\rangle$, then we obtain our result.

For suppose that $G=H \times\{1, q\}$ where $H$ is a subgroup of index 2 of $G$. Then $W(F)=S[\{\langle 1\rangle, q\}]$ with $S=R[H]$. We claim that $W(F)=S\left[\left\{\langle 1\rangle,\left\langle b_{q}\right\rangle\right\}\right]$, which is what is necessary to complete the proof as in 4.3. Recalling the proof of 4.1, it suffices to show that 
$\left\langle b_{q}\right\rangle=r+s q$ with $r, s \in S$ and $s$ odd-dimensional, i.e., to show that $\tau_{q}\left(b_{q}\right)=1$.

Now for each $i=1, \cdots, k$, write $\left\langle b_{i}\right\rangle \varphi_{i}=r_{i}+s_{\imath} q$ with $r_{\imath}, s_{i} \in$ $S$. Then for all $i$,

$$
\operatorname{dim}_{0}\left(s_{\imath}\right) \equiv\left(\operatorname{dim} \varphi_{i}\right) \tau_{q}\left(b_{i}\right)(\bmod 2),
$$

since $\varphi_{i} \in T(F)$ may be diagonalized over $A(F)$ and $\tau_{q}\left(a b_{\imath}\right)=\tau_{q}\left(b_{i}\right)$ for any $a \in A(F) \subseteq \operatorname{ker} \tau_{q}$. But for $i \geqq 2, \operatorname{dim} \varphi_{i}$ is even, so for such $i, \operatorname{dim}_{0}\left(s_{\imath}\right)=0$. Finally,

$$
q=\sum_{\imath=1}^{k} r_{i}+q \sum_{i=1}^{k} s_{\imath},
$$

so $\sum_{v=1}^{k} s_{i}=1$. Hence $\operatorname{dim}_{0}\left(s_{1}\right)$ must be odd, so not only is $\operatorname{dim} \varphi_{1}$ odd, but also $\tau_{q}\left(b_{q}\right)=1$.

\section{REFERENCES}

1. L. Berman, Pythagorean fields and the Kaplansky radical, J. Algebra, 61 (1979), 497-507.

2. L. Berman, C. Cordes and R. Ware, Quadratic forms, rigid elements, and power series fields, to appear J. Algebra.

3. H. Gross and H. R. Fischer, Non-real fields $k$ and infinite dimensional $k$-vector spaces (Quadratic forms and linear topologies, II), Math. Ann., 159 (1965), 285-308.

4. T. Y. Lam, The Algebraic Theory of Quadratic Forms, W. A. Benjamin, Reading, Ma., 1973.

5. T. A. Springer, Quadratic forms over fields with a discrete valuation, Indag. Math., 17 (1955), 352-362.

Received June 11, 1979.

UNIVERSITY OF OKLAHOMA

NORMAN, OK 73019 



\section{PACIFIC JOURNAL OF MATHEMATICS}

\section{EDITORS}

DONALD BABBITT (Managing Editor)

University of California

Los Angeles, CA 90024

HUGo RossI

University of Utah

Salt Lake City, UT 84112

C. C. MOORE and ANDREW OGG

University of California

Berkeley, CA 94720
J. DugundJI

Department of Mathematics

University of Southern California

Los Angeles, CA 90007

R. FinN and J. Milgram

Stanford University

Stanford, CA 94305

ASSOCIATE EDITORS
E. F. BECKENBACH
B. H. NeUmanN
F. WOLF
K. YoSHIDA

\section{SUPPORTING INSTITUTIONS}

UNIVERSITY OF BRITISH COLUMBIA

CALIFORNIA INSTITUTE OF TECHNOLOGY

UNIVERSITY OF CALIFORNIA

MONTANA STATE UNIVERSITY

UNIVERSITY OF NEVADA, RENO

NEW MEXICO STATE UNIVERSITY

OREGON STATE UNIVERSITY

UNIVERSITY OF OREGON
UNIVERSITY OF SOUTHERN CALIFORNIA

STANFORD UNIVERSITY

UNIVERSITY OF HAWAII

UNIVERSITY OF TOKYO

UNIVERSITY OF UTAH

WASHINGTON STATE UNIVERSITY

UNIVERSITY OF WASHINGTON

The Supporting Institutions listed above contribute to the cost of publication of this Journal, but they are not owners or publishers and have no responsibility for its content or policies.

Mathematical papers intended for publication in the Pacific Journal of Mathematics should be in typed form or offset-reproduced, (not dittoed), double spaced with large margins. Please do not use built up fractions in the text of the manuscript. However, you may use them in the displayed equations. Underline Greek letters in red, German in green, and script in blue. The first paragraph or two must be capable of being used separately as a synopsis of the entire paper. Please propose a heading for the odd numbered pages of less than 35 characters. Manuscripts, in triplicate, may be sent to any one of the editors. Please classify according to the scheme of Math. Reviews, Index to Vol. 39. Supply name and address of author to whom proofs should be sent. All other communications should be addressed to the managing editor, or Elaine Barth, University of California, Los Angeles, California, 90024.

50 reprints to each author are provided free for each article, only if page charges have been substantially paid. Additional copies may be obtained at cost in multiples of 50 .

The Pacific Journal of Mathematics is issued monthly as of January 1966. Regular subscription rate: $\$ 84.00$ a year (6 Vols., 12 issues). Special rato: $\$ 42.00$ a year to individual members of supporting institutions.

Subscriptions, orders for numbers issued in the last three calendar years, and changes of address shoud be sent to Pacific Journal of Mathematics, P.O. Box 969, Carmel Valley, CA 93924, U.S.A Old back numbers obtainable from Kraus Periodicals Co., Route 100, Millwood, NY 10546.

PUBLISHED BY PACIFIC JOURNAL OF MATHEMATICS, A NON-PROFIT CORPORATION

Printed at Kokusai Bunken Insatsusha (International Academic Printing Co., Ltd.). 8-8, 3-chome, Takadanobaba, Shinjuku-ku, Tokyo 160, Japan.

Copyright (C) 1980 by Pacific Jounal of Mathematics Manufactured and first issued in Japan 


\section{Pacific Journal of Mathematics \\ Vol. 89, No. $2 \quad$ June, 1980}

Frank Hayne Beatrous, Jr. and R. Michael Range, On holomorphic

approximation in weakly pseudoconvex domains................. 249

Lawrence Victor Berman, Quadratic forms and power series fields ...... 257

John Bligh Conway and Wacław Szymański, Singly generated antisymmetric operator algebras ....................... 269

Patrick C. Endicott and J. Wolfgang Smith, A homology spectral sequence for submersions . . . .................................

Sushil Jajodia, Homotopy classification of lens spaces for one-relator groups with torsion ................................ 301

Herbert Meyer Kamowitz, Compact endomorphisms of Banach

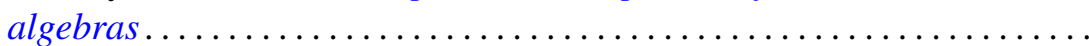

Keith Milo Kendig, Moiré phenomena in algebraic geometry: polynomial

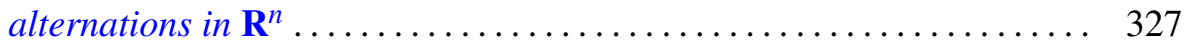

Cecelia Laurie, Invariant subspace lattices and compact operators....... 351

Ronald Leslie Lipsman, Restrictions of principal series to a real form . . . . . 367

Douglas C. McMahon and Louis Jack Nachman, An intrinsic

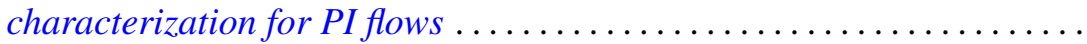

Norman R. Reilly, Modular sublattices of the lattice of varieties of inverse semigroups .................................... 405

Jeffrey Arthur Rosoff, Effective divisor classes and blowings-up of $\mathbf{P}^{2}$ 419

Zalman Rubinstein, Solution of the middle coefficient problem for certain

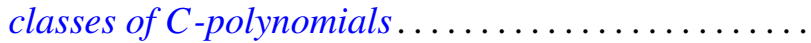

Alladi Sitaram, An analogue of the Wiener-Tauberian theorem for spherical transforms on semisimple Lie groups ................

Hal Leslie Smith, A note on disconjugacy for second order systems ...

J. Wolfgang Smith, Fiber homology and orientability of maps ...

Audrey Anne Terras, Integral formulas and integral tests for series of positive matrices. 